\title{
ESTIMACIÓN DEL ÍNDICE DE ÁREA FOLIAR EN LA RESERVA DE LA BIÓSFERA MARIPOSA MONARCA
}

\section{LEAF AREA INDEX ESTIMATION IN THE MONARCH BUTTERFLY BIOSPHERE RESERVE}

\author{
Silvia Velasco López ${ }^{1}$, Omar Champo Jiménez ${ }^{2}$, Ma. Luisa España Boquera ${ }^{2 *}$ y Frédéric Baret ${ }^{3}$
}

\begin{abstract}
${ }^{1}$ Facultad de Biología, Universidad Michoacana de San Nicolás de Hidalgo (UMSNH), Ciudad Universitaria, Edificio R. 58880, Morelia, Michoacán, México. ${ }^{2}$ Instituto de Investigaciones Agropecuarias y Forestales, UMSNH. Posta Veterinaria y Zootécnica, km 9.5 carretera Morelia-Zinapécuaro, Michoacán, México. ${ }^{3}$ Institut National de la Recherche Agronomique, Site AGROPARC. 84914, Avignon, France.
\end{abstract}

* Autor para correspondencia (boquera@umich.mx)

\section{RESUMEN}

El índice de área foliar es la principal variable biofísica utilizada para modelar muchos procesos vegetales. En el presente trabajo se propone un método para obtener un mapa del índice de área foliar en la Reserva de la Biósfera Mariposa Monarca, basado en el ajuste de una función de transferencia radiativa entre estimaciones del índice de área foliar obtenidas en unos pocos puntos de muestreo con fotografías hemisféricas y los datos radiométricos correspondientes de una imagen del satélite SPOT (satellite pour l'observation de la Terre). Previo al ajuste de la función de transferencia, los datos fueron corregidos del efecto del relieve. Se eligió una combinación lineal de bandas de la imagen SPOT $(r=0.608, R M S E=0.493)$. La aplicación de la función de transferencia a la imagen de la reserva permitió construir un mapa de índice de área foliar y evaluar el estado del bosque en una fecha cercana a la declaración de reserva de la biósfera. Este trabajo se llevó a cabo en el marco del proyecto internacional VALERI (validation of european remote sensing instruments).

Palabras clave: Índice de área foliar, función de transferencia, VALERI.

\section{SUMMARY}

Leaf area index is the main biophysical variable used to model many plant processes. In this work a method is proposed to obtain a leaf area index map of the Monarch Butterfly Biosphere Reserve based in the adjustment of a radiative transfer function between the leaf area index, estimated in some sample points using hemispherical photographs, and the corresponding radiometrical data of a SPOT (satellite pour l'observation de la Terre) image. Before the adjustment of the radiative transfer function, data were corrected with respect to the slope effect. In order to adjust a function, several vegetation indices were calculated and linear and non linear functions were tested, and a linear combination of SPOT bands was chosen $(r=0.608$, RMSE $=0.493)$. The transfer function was applied to the forest image corresponding to the Monarch Butterfly Reserve to obtain a leaf area index map of a date near the declaration of biosphere reserve. This work was performed in the context of the VALERI (validation of european remote sensing instruments) international project.
Index words: Leaf area index, transfer function, VALERI.

\section{INTRODUCCIÓN}

El índice de área foliar se define generalmente como el área de una cara (el haz) de tejido fotosintético por unidad de superficie horizontal de suelo (Myneni et al., 2002) y representa el tamaño de la interfase planta-atmósfera, por lo que es la principal variable utilizada para modelar fotosíntesis y evapotranspiración, evaluar la radiación fotosintéticamente activa absorbida (fAPAR) y describir el microclima de la planta (Weiss et al., 2004; Jonckheere et $a l ., 2004)$.

Los métodos directos utilizados para medir el índice de área foliar resultan costosos, tediosos y destructivos, por lo que no son útiles para una aplicación a gran escala. Hay métodos indirectos que estiman el índice de área foliar a partir de otra variable, más fácil de medir (Jonckheere et al., 2004), como la transmisión de la luz a través del dosel en términos de fracción de huecos, que utilizan equipos como el SunScan ceptometer ${ }^{\circledR}$ (Delta-T, UK), AccuPAR ${ }^{\circledR}$ (Decagon, UK), Demon ${ }^{\circledR}$ (CSIRO, Australia), LAI2000 Plant Canopy Analyser ${ }^{\circledR}$ (Li-Cor, USA) y las fotografías hemisféricas (tomadas con lentes tipo "ojo de pescado"). También es posible hacer estimaciones mediante relaciones alométricas entre el índice de área foliar y otra dimensión más fácil de obtener, como altura del tronco o anchura de copa, etc.; sin embargo, estas relaciones deben ser definidas para cada especie y situación particular (Holdaway et al., 2008; Macfarlane et al., 2007; Rautiainen, 2005). En todo caso, la estimación a mediana o gran escala del índice de área foliar con mediciones de campo puede ser inviable, por lo que una 
opción puede ser el apoyo en imágenes satelitales de teledetección.

Desde el año 2000, con el lanzamiento de captores como MODIS (moderate resolution imaging spectroradiometer) (Terra y Aqua), VEGETATION o MERIS (medium resolution image spectroradiometer instrument), de mediana resolución espacial y alta temporal, las propias agencias espaciales han empezado a generar mapas de índice de área foliar, a veces disponibles en internet. Así, la NASA (National Aeronautics and Space Administration) ofrece productos de índice de área foliar y fAPAR (fraction of absorbed photosyntheticaly active radiation) generados con imágenes MODIS para un periodo de $8 \mathrm{~d}$ a $1 \mathrm{~km}$ de resolución espacial (Shabanov et al., 2005, Huang et al., 2006; Yang et al., 2006). Estos productos dan continuidad a los generados mensualmente a partir de imágenes AVHRR (advanced very high resolution radiometer) con resolución de $16 \mathrm{~km}$. Por su parte, la ESA (European Space Agency), en el marco del proyecto GLOBCARBON, está produciendo mapas globales de índice de área foliar a partir de imágenes VEGETATION, MERIS, AATSR (advanced along track scanning radiometer) y AVHRR. Asimismo, el programa CYCLOPES (carbon cycle and change in land observational products from an ensemble of satellites) de la Comisión Europea, ha desarrollado mapas de índice de área foliar a partir de datos de múltiples sensores (Baret et al., 2007; Weiss et al., 2007; Verger et al., 2008).

La generación de productos satelitales similares por diferentes agencias espaciales ha impulsado el desarrollo de los respectivos programas de validación (Morrisette et al., 2006, Pandya et al., 2006, Abuelgasim et al., 2006). En este contexto, en el año 2001 se inició el proyecto VALERI (validation of european remote sensing instruments) (Baret et al., 2007, en línea), con el objetivo de generar una metodología efectiva para construir mapas de índice de área foliar con alta resolución espacial para la validación de productos globales de moderada resolución. Para la primera fase del proyecto VALERI, concluida en 2009, se establecieron más de 30 sitios de muestreo en el mundo, entre los que se encuentra la Reserva de la Biósfera Mariposa Monarca. En el presente trabajo se expone la metodología generada para establecer mapas de índice de área foliar en esta reserva, con las adaptaciones realizadas para corregir el efecto de la pendiente del terreno. Esta metodología se aplica para la obtención de un mapa de índice de área foliar de la reserva correspondiente a 2001, sobre el que se establece el porcentaje de bosque y de deforestación para esta fecha.

\section{MATERIALES Y MÉTODOS}

La Reserva de la Biósfera Mariposa Monarca (Danaus plexippus plexippus L.) es una zona prioritaria para la conservación en México (Figura 1), pues en ella se presenta el fenómeno natural de su migración. Sin embargo, las extracciones clandestinas de madera han tenido un fuerte impacto en la condición del bosque. La vegetación es predominantemente bosque de oyamel (Abies religiosa L.). El relieve es muy irregular, con pendientes de hasta $45^{\circ}$.

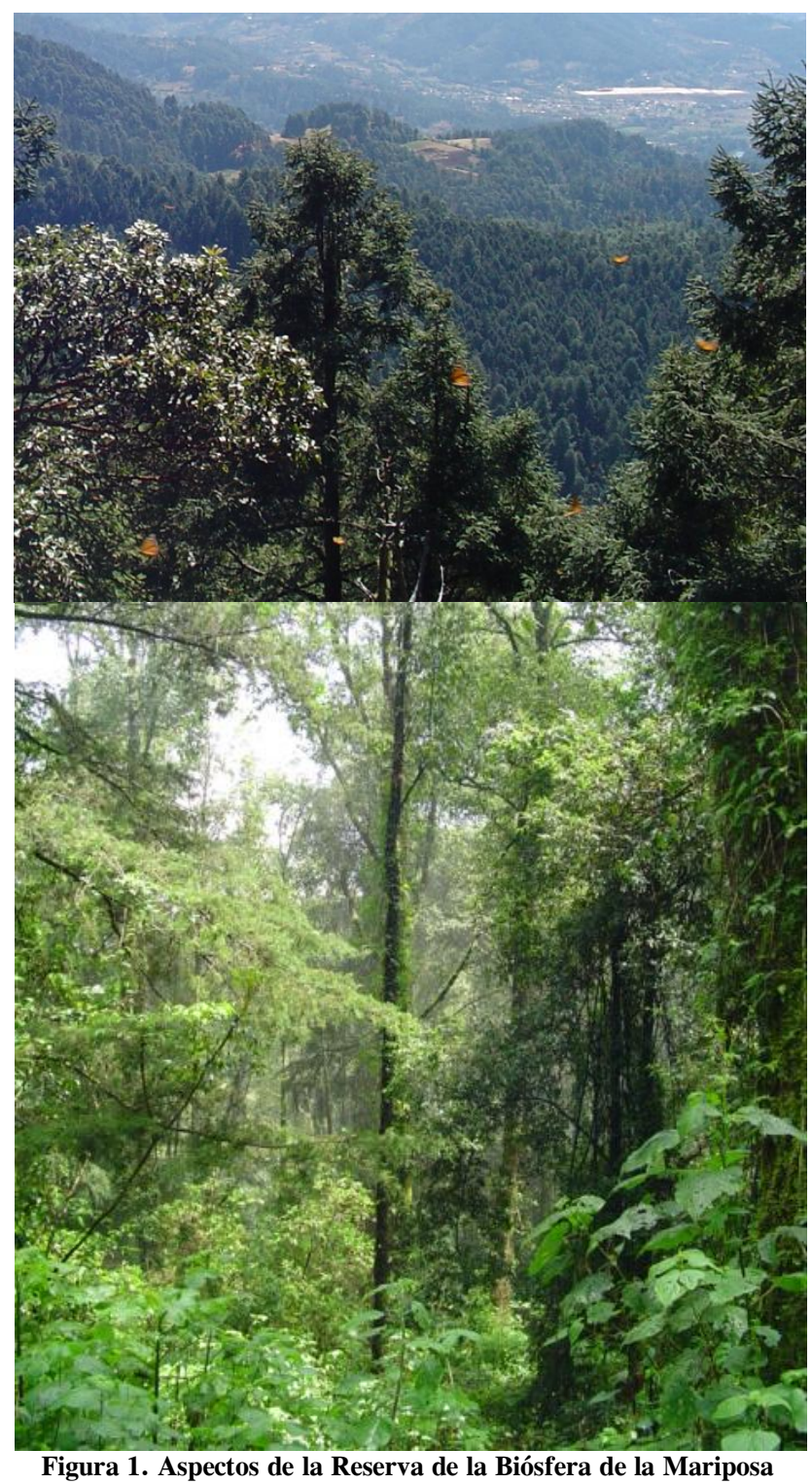
Monarca.

Conforme al protocolo VALERI, en la reserva se eligió un área de $3 \mathrm{~km} \times 3 \mathrm{~km}$ que se dividió en nueve partes iguales de $1 \mathrm{~km}^{2}$. En cada una se tomaron al azar un mínimo de tres puntos de muestreo, llamados ESU 
(elementary simple unit), que corresponden a una superficie de $20 \mathrm{~m}$ × $20 \mathrm{~m}$, equivalente a un píxel SPOT. En cada ESU, que es georreferenciado con un instrumento GPS (global positionning system), se tomaron 12 medidas individuales de índice de área foliar, mediante fotografías hemisféricas hacia el cielo (up) y hacia el suelo (down). El análisis de las fotografías hemisféricas se hizo con el programa CAN-EYE (Baret et al., 2007, en línea). El índice de área foliar de cada ESU es el promedio de los 12 valores de índice de área foliar, up más down. En general no es necesario tomar en cuenta el efecto de la inclinación del terreno sobre la estimación del índice de área foliar con fotografías hemisféricas (España et al., 2008). Para el presente trabajo, la campaña de muestreo se llevó a cabo en diciembre de 2001, en un total de 30 puntos de muestreo.

Los datos radiométricos de reflectancia (relación entre la luz reflejada y la incidente en función de la longitud de onda) de cada ESU se obtuvieron de una imagen satelital SPOT (satellite pour l'observation de la Terre), tomada en la fecha más cercana posible a la del muestreo. Para este trabajo se adquirió una imagen de octubre de 2001, ya que para ese año no existen en el catálogo imágenes de noviembre y diciembre. La imagen se corrigió geométrica y geográficamente con respecto a un mapa INEGI (Instituto Nacional de Estadística, Geografía e Informática de México) de la zona, y se utilizaron 20 puntos de control (probabilidad de error de $0.99 \%$ ). Los datos numéricos (digital numbers) se calibraron con respecto a la ganancia de los sensores y se aplicó una corrección atmosférica.

Los datos de reflectancia se corrigieron del efecto del relieve, considerando la radiación directa y difusa y aplicando el método mejorado $(\mathrm{SPS}+\mathrm{C})$ basado en las correcciones-C de Meyer (Teillet et al., 1982; Meyer et al., 1993), propuesto por Soenen (Soenen et al., 2005) y utilizado por otros autores como Heiskanen (2006). A partir de las reflectancias corregidas se calcularon los índices de vegetación que se muestran en el Cuadro 1.

Cuadro 1. Índices de vegetación utilizados en el ajuste de los modelos. b1: banda verde de la imagen SPOT $(540 \mathrm{~nm})$; b2: banda roja $(650 \mathrm{~nm})$; b3: banda del infrarrojo cercano (NIR, $835 \mathrm{~nm})$; y b4 banda del infrarrojo medio (SWIR, $1630 \mathrm{~nm}$ ).

\begin{tabular}{|c|c|c|}
\hline Índice & Descripción & Ecuación \\
\hline SR & Simple ratio & $\mathrm{b} 3 / \mathrm{b} 2$ \\
\hline $\mathrm{RN}$ & Red NIR index & $\mathrm{b} 3 * \mathrm{~b} 2$ \\
\hline NDVI & $\begin{array}{l}\text { Normalized difference } \\
\text { vegetation index }\end{array}$ & $(\mathrm{b} 3-\mathrm{b} 2) /(\mathrm{b} 3+\mathrm{b} 2)$ \\
\hline DVI & Difference vegetation index & $\mathrm{b} 3-\mathrm{b} 2$ \\
\hline RDVI & Red difference vegetation index & $\sqrt{ }(\mathrm{NDVI} * \mathrm{DVI})$ \\
\hline (Chl)RIgreen & Chlorophylle ratio index, green & $(1 / \mathrm{b} 1-1 / \mathrm{b} 3) * \mathrm{~b} 3$ \\
\hline
\end{tabular}

Con $2 / 3$ de los ESU se calcularon regresiones múltiples entre los niveles de reflectancia corregidos o los índices de vegetación calculados, y el índice de área foliar medido en campo. La precisión del ajuste de las curvas se calculó por el coeficiente de determinación, $\mathrm{R}^{2}$. El error asociado al ajuste se calculó como la raíz del error cuadrático medio ponderado (PRMSE) con respecto a la distancia de cada punto a la recta de regresión (los residuos de la regresión). Con el fin de evaluar el error asociado a la elección de los ESUs utilizados para el ajuste, el proceso se repitió de manera iterativa, donde el conjunto de ESUs se dividió en tres particiones y, tomando cada vez dos, se ajustaron las curvas y se calculó el error cuadrático medio (RMSE). El RMSE cruzado (CRMSE) es el promedio de los errores cuadráticos de las tres interacciones. Con el resto de los puntos $(1 / 3$ de los ESU) se evaluó la capacidad de las fórmulas ajustadas para estimar el índice de área foliar, con base en la correlación (r) entre los valores estimados y los valores observados. Los errores asociados con la estimación de valores de índice de área foliar se calcularon como medidos el RMSE. Todos los cálculos se efectuaron con la función robustfit que es una herramienta de estadística del programa de cálculos matemáticos Matlab\&Simulink ${ }^{\circledR}$ (The MathWorks) (versión 8.0).

La relación matemática que presenta el menor error ponderado (PRMSE) y un buen coeficiente de determinación $\left(\mathrm{R}^{2}\right)$, además de presentar la correlación más alta (r) en la validación, se escogió como función de transferencia radiativa, siempre y cuando la regresión pasara por el origen. Esta función se aplicó a cada píxel de la imagen de la zona considerada para generar un mapa de índice de área foliar.

\section{RESULTADOS Y DISCUSIÓN}

El sitio de muestreo está definido por los vértices de coordenadas UTM 14Q 3640E $21775 \mathrm{~N}$ y $3672 \mathrm{E} 21745 \mathrm{~N}$. Se consideró un área de un poco más de los $9 \mathrm{~km}^{2}$ indicados en el protocolo porque algunos puntos de muestreo se tomaron muy cerca de los bordes del área inicialmente definida.

El Cuadro 2 muestra las combinaciones de bandas e índices que proporcionaron un mejor ajuste de los valores de índice de área foliar medidos en campo, al considerar $2 / 3$ de los ESUs. Las combinaciones (b2, b4, RN), (b1, b3, b4, RN) y (b1, b2, b3, b4) (siglas según Cuadro 1) fueron las que presentaron un mejor coeficiente de determinación $\left(\mathrm{R}^{2}\right)$, que en ningún caso llegó a 0.5 , con errores ponderados y cruzados relativamente bajos. 
Cuadro 2. Combinaciones de bandas e índices (según Cuadro 1) que en la regresión múltiple permiten el mejor ajuste con los valores del índice de área foliar con $2 / 3$ de los puntos de muestreo. $\mathbf{R}^{2}$ : coeficiente de determinación al ajuste; PRMSE: raíz del error cuadrático medio ponderado; CRMSE: raíz del error cuadrático medio cruzado.

\begin{tabular}{lccc}
\hline Combinación de bandas & $\mathrm{R}^{2}$ & PRMSE & CRMSE \\
\hline b2 & 0.118 & 0.593 & 0.616 \\
SR & 0.343 & 0.501 & 0.559 \\
NDVI & 0.338 & 0.504 & 0.552 \\
RDVI & 0.284 & 0.513 & 0.616 \\
(Ch1)RIgreen & 0.303 & 0.526 & 0.557 \\
b3,b4,RN & 0.396 & 0.504 & 0.557 \\
b2,b4,RN & 0.439 & 0.502 & 0.531 \\
b1,b4,RN & 0.156 & 0.608 & 0.635 \\
b2,b3,b4 & 0.397 & 0.511 & 0.546 \\
b3,b4, (Chl)RIgreen & 0.398 & 0.517 & 0.548 \\
b1,b3,b4,RN & 0.420 & 0.525 & 0.554 \\
b1,b2,b3,b4 & 0.411 & 0.530 & 0.553 \\
\hline
\end{tabular}
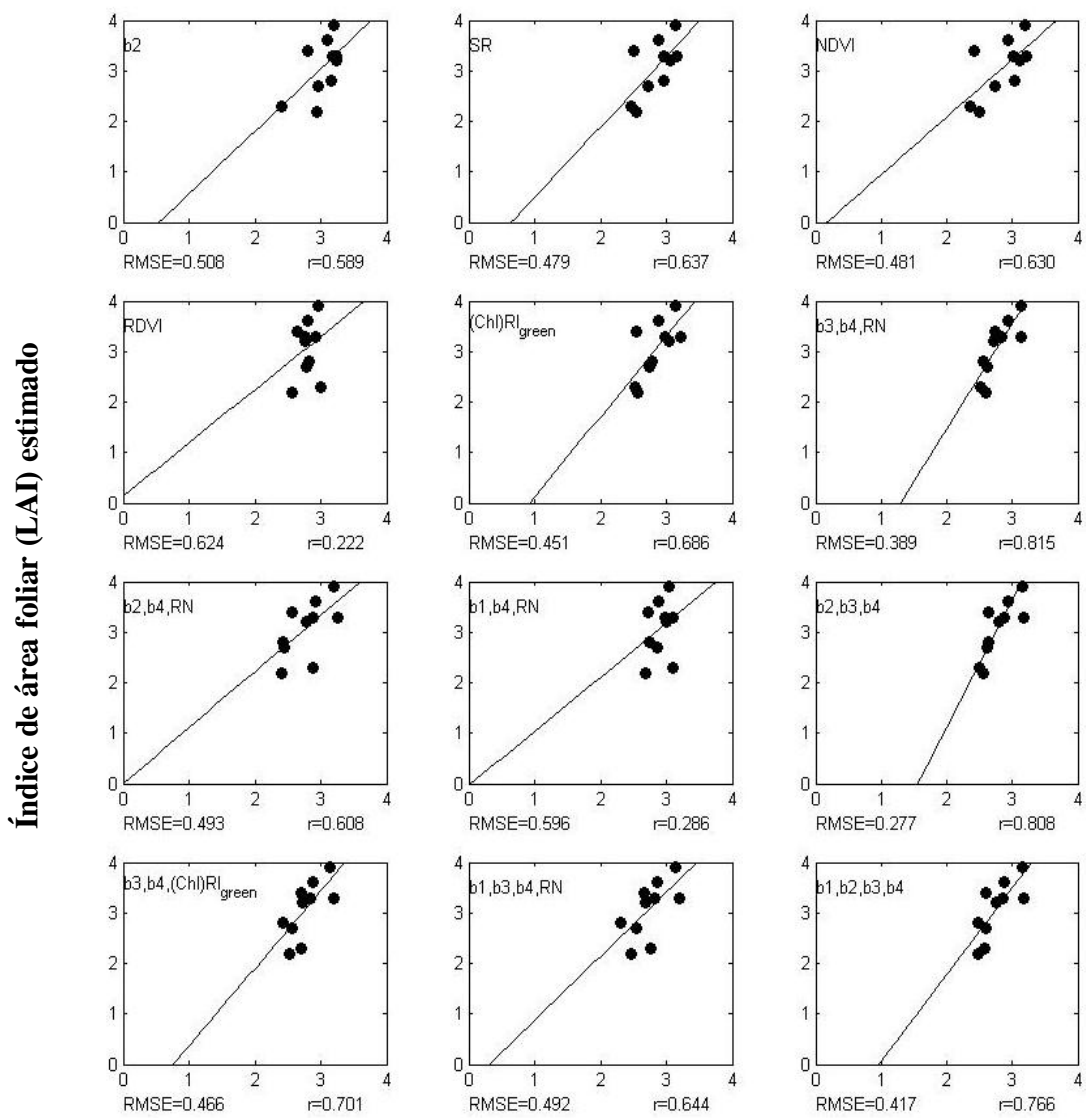

Índice de área foliar (LAI) medido

Figura 2. Regresiones de diferentes combinaciones de bandas de la imagen SPOT. b1: verde, b2: rojo, b3: infrarrojo cercano (NIR), b4: infrarrojo medio (SWIR).

La función de transferencia se aplicó a toda la imagen del sitio VALERI para obtener el mapa de índice de área foliar (Figura 2). A los píxeles correspondientes a los claros se les dio el valor de índice de área foliar mínimo (0.1). En las zonas arboladas se pueden distinguir diferentes niveles de índice de área foliar, que se traducen en diferentes densidades de vegetación (representadas en rojo, amarillo y verde). Los valores de índice de área foliar calculados (entre 0 y 4.5) son comparables a los obtenidos en los demás sitios de muestreo del proyecto VALERI, que corresponden a bosques y en general se encuentran en zonas planas o con pendientes suaves. En la 
mayoría de estudios se han utilizado funciones de transferencia radiativa que resultan de regresiones entre el índice de área foliar y las diferentes bandas de la imagen SPOT, o una relación entre ellas. En pocos casos han dado mejores ajustes las regresiones con logaritmos. Los errores reportados de las estimaciones (RMSE) fluctuaron entre 0.1 a 1 , en función del número de puntos de muestreo, que varió de 20 a 80 , según los sitios, y se ha reportado que 30 es el número óptimo de ESU en zonas relativamente homogéneas.
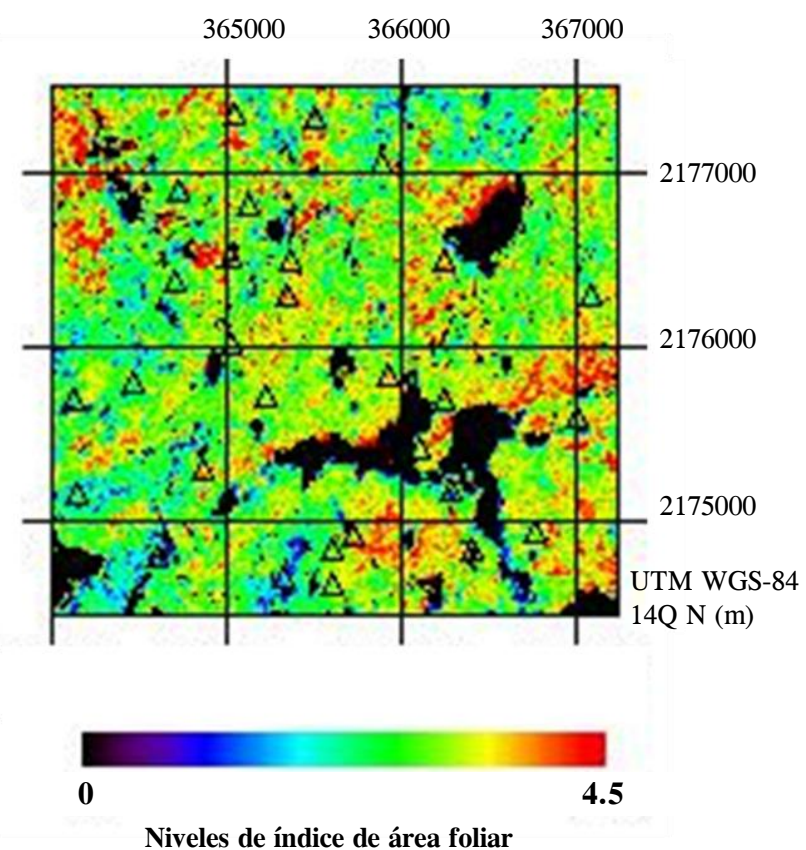

Figura 2. Mapa de índice de área foliar de la zona de estudio. Negro: pastos; azul: zona deforestada; verde, amarillo, rojo: bosque poco a muy denso.

Finalmente, la función de transferencia se aplicó a la imagen de toda la Reserva de la Mariposa Monarca, para obtener el mapa de índice de área foliar correspondiente a finales de 2001 (Figura 3), sin considerar zonas agrícolas (en negro en la figura). Como en la imagen de la zona VALERI, a los claros se les asignó un valor de índice de área foliar bajo (en negro en la figura). La aplicación de la ecuación de transferencia pone de relieve las zonas deforestadas (en azul en Figura 3).

A partir de este mapa se puede aproximar que, en el momento de la declaración de Reserva de la Biósfera, cerca de $45 \%$ de la superficie total (54 243 ha) sin considerar Altamirano de la Reserva de la Mariposa Monarca estaba ocupado por terrenos agrícolas y claros (índice de área foliar inferior a 1), mientras que la superficie total arbolada se repartía casi en proporciones iguales entre bosque más (27 \% con índice de área foliar superior a 3) y menos denso (28\% con índice de área foliar entre 1 y 3 ). La zona núcleo no presentaba agricultura, pero sí problemas de deforestación.

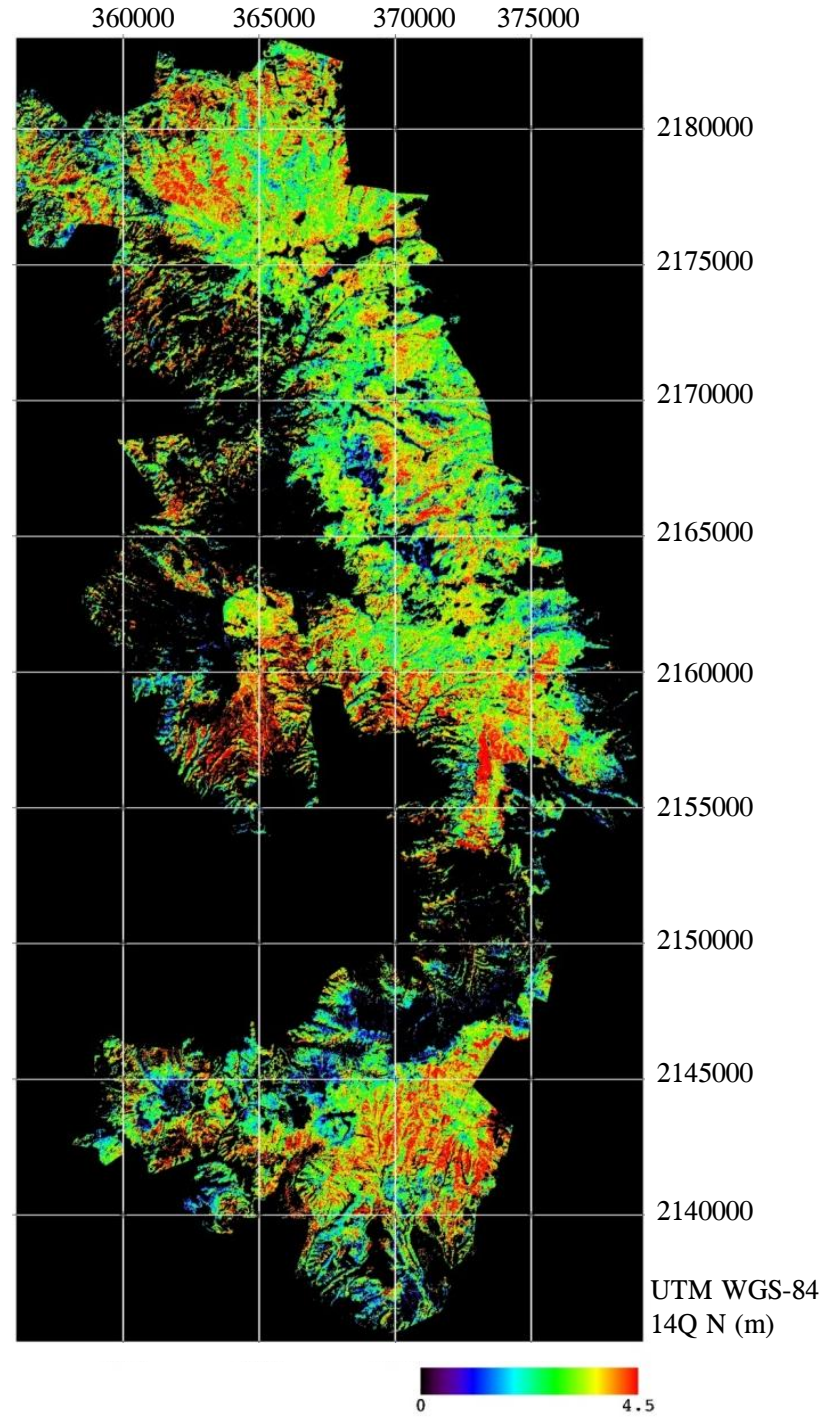

Niveles de índice de área foliar

Figura 3. Mapa de índice de área foliar de la reserva de la Mariposa Monarca en 2001. Negro: pastos y zonas agrícolas; azul: zona deforestada; verde, amarillo, rojo: bosque poco a muy denso.

\section{AGRADECIMIENTOS}

A Raúl Cárdenas Navarro, Luís López Pérez y Jean Francois Hanocq por su valiosa participación en los muestreos de campo, y a Marie Weiss por su asesoría en la utilización del programa CAN-EYE. Este trabajo se realizó con el financiamiento de SEP-PROMEP y la Coordinación de la Investigación Científica de la Universidad Michoacana de San Nicolás de Hidalgo. 


\section{BIBLIOGRAFÍA}

Abuelgasim A, R Fernandes, S Leblanc (2006) Evaluation of national and global LAI products derived from optical remote sensing instruments over Canada. IEEE Trans. Geosci. Remote Sens. 44:1872-1884.

Baret F, M Weiss, M Leroy, D Allard, S Garrigues, H Bohbot, R Bosseno, M España, V Gond, P Guinot, D Guyon, C Lelong, E Mougin, $T$ Nilson, $R$ Vintilla (Marzo 2007). VALERI: validation of land satellite product. Disponible en: www.avignon.inra.fr/valeri

Baret F, O Hagolle, B Geiger, P Bicheron, B Miras, M Huc, B Betherlot, F Niño, M Weiss, O Samain, J L Roujean, M Leroy (2007) LAI, fAPAR and fCover CYCLOPES global products derived from VEGETATION. Part 1: Principles of the Algorithm. Remote Sens. Environ. 110:275-286.

España M L, F Baret, M Weiss (2008) Slope correction for LAI estimation from gap fraction measurements. Agric. For. Meteorol. 148:1553-1562.

Heiskanen J (2006) Estimating aboveground tree biomass and leaf area index in a mountain birch forest using ASTER satellite data. Int. J. Rem. Sens. 27:1135-1158.

Holdaway R J, R B Allen, P W Clinton, M R Davis, D A Coomes (2008) Intraspecific changes in forest canopy allometries during self-thinning. Func. Ecol. 22:460-469.

Houborg R M Anderson, C Daughtry (2009) Utility of an image-based canopy reflectance modelling tool for remote estimation of LAI and leaf chlorophyll content at the field scale. Remote Sens. Environ. 113:259-274.

Huang D, W Yang, Bin Tan, M Rautiainen, Z Ping, J Hu, N Shabanov, S Linder, Y Knyazikhin, R Myneni (2006) The importance of measurement errors for deriving accurate reference leaf area index maps for validation of moderate resolution satellite LAI products. IEEE Trans. Geosci. Remote Sens. 44:1866-1871

Jonckheere I, S Fleck, K Nacckaerts, B Muys, P Coppin, M Weiss, F Baret (2004) Review of methods for in situ leaf area index determination. Part I: Theories, sensors and hemispherical photography. Agric. For. Meteorol. 121:19-35.

Macfarlane C, M Hoffman, D Eamus, $N$ Kerp, $S$ Higginson, $R$ McMurtrie, M Adams (2007) Estimation of leaf area index in eucalypt forest using digital photography. Agric. For. Meteorol. 143:176-188.

McAllister D M, C Valeo (2007) A robust new method for the remote estimation of LAI in montane and boreal forest. Int. J. Remote. Sens. 28:1891-1905.

Meyer P, K Itten, T Kellenberger, S Sandmeier, R Sandmeier (1993) Radiometric corrections of topographically induced effects on Landsat TM data in an alpine environment. ISPRS J. Photogramm. Remote Sens. 48:17-28.

Morrisette T J, F Baret, L Privette, R Myneni, J Nickeson, S Garrigues, N Shabanov, M Weiss, R Fernandes, S Leblanc,
M Kalacska, G Sánchez-Azofeifa, M Chubey, B Rivard, P Stenberg, M Rautiainen, P Voipio, T Manninen, A Pilant, T Lewis, J Iiames, R Colombo, M Meroni, L Busetto, W Cohen, D Turner, E Warner, G Petersen, G Seufert, R Cook (2006) Validation of global moderate resolution LAI products : a framework proposed within the CEOS land product validation subgroup. IEEE Trans. Geosci. Remote Sens. 44:1804-1817.

Myneni R, S Hoffman, Y Knyazikhin, JL Privette, J Glassy, Y Tian, Y Wang, X Song, Y Zhang, G Smith, A Lotsch, M Friedl, J T Morisette, P Votava, R Nemani, S W Running (2002) Global products of vegetation leaf area and fraction absorbed PAR from year one of MODIS data. Remote Sens. Environ. 83:214-231.

Pandya M, R Singh, K Chaudhari, G Bairagi, R Sharma, V Dadhwal, J Singh Parihar (2006) Leaf area index retrieval using IRS LISS- III sensor data and validation of the MODIS LAI product over central India. IEEE Trans. Geosci. Remote Sens. 44:1858-1865

Rautiainen M (2005) Retrieval of leaf area index for a coniferous forest by inverting a forest reflectance model. Remote Sens. Environ. 99:295-303.

Shabanov N, D Huang, W Yang, B Tan, Y Knyazikhin, R Myneni, D Ahl, S Gower, A Huete, L Aragao, Y Shimabukuro (2005) Analysis and optimization of the MODIS leaf area index algorithm retrievals over broadleaf forest. IEEE Trans. Geosci. Remote Sens. 43:1855-1865.

Soenen S A, D R Peddle, C A Coburn (2005) SCS + C: A modified sucanopy-sensor topographic correction in forested terrain. IEEE Trans. Geosci. Remote Sens. 43:2148-2159.

Teillet P M, B Guindon, D Goodenough (1982) On the slope-aspect correction of multispectral scanner data. Can. J. Remote Sens. 8:84-106.

Verger A, Baret F, Weiss M (2008) Performances of neuronal networks for deriving LAI estimates from existing CYCLOPES and MODIS products. Remote Sens. Environ. 112:2789-2803.

Weiss M, F Baret, G Smith, I Jonckheere, P Coppin (2004) Review of methods for in situ leaf area index determination, part II: Estimation of LAI, errors and sampling. Agric. For Meteorol. 121:37-53

Weiss M, F Baret, S Garrigues, R Lacaze (2007) LAI, fAPAR and fCover CYCLOPES global products derived from VEGETATION. Part 2: Validation and comparison with MODIS collection 4 products. Remote Sens. Environ. 110:317331.

Yang W, B Tan, D Huang, M Rautiainen, N Shabanov, Y Wang, J Privette, K Huemmrich, Fensholt, I Sandholt, M Weiss, D Ahl, S Gower, R Nemani, Y Knyazikhin, R Myneni (2006) MODIS leaf area index products: From validation to algorithm improvement. IEEE Trans. Geosci. Remote Sens. 44:18851898. 